\title{
CULTURA DE SEGURIDAD DEL PACIENTE POR PERSONAL DE ENFERMERÍA EN BOGOTÁ, COLOMBIA
}

\section{PATIENT SAFETY CULTURE OF NURSING STAFF IN BOGOTÁ, COLOMBIA}

\author{
Olga Gómez Ramírez ${ }^{*}$ \\ Wendy Arenas GutiérRez ${ }^{* *}$ \\ Lizeth GonZÁleZ VEGA ${ }^{* * *}$ \\ JenNifer Garzón Salamanca ${ }^{* * * *}$ \\ ERIKa Mateus Galeano ${ }^{* * * * *}$ \\ Amparo Soto GÁmEZ ${ }^{* * * * *}$
}

\begin{abstract}
RESUMEN
La cultura de seguridad del paciente está relacionada con las creencias y actitudes que asumen las personas en su práctica para garantizar que no experimentará daño innecesario o potencial asociado a la atención en salud. Este estudio describe la percepción de dicha cultura referida por el personal de enfermería en instituciones prestadoras de servicios de salud del tercer nivel de complejidad que hacen parte del Sistema de Seguridad Social en Salud en Bogotá, Colombia. Método: Estudio descriptivo, transversal, exploratorio realizado durante el 2009. La muestra estuvo constituida por 201 profesionales y técnicos en enfermería que aceptaron participar voluntariamente y cumplieron los requisitos de inclusión planteados. La medición se realizó con el instrumento Hospital Survey on Patient Safety Culture. El cuestionario fue autoadministrado después de informar el propósito y cumplir las consideraciones éticas. Resultados: La cultura de seguridad del paciente referida por el personal de enfermería se evidencia por resultados positivos en la coordinación y comunicación eficaz dentro de las unidades y la percepción generalizada de que la gerencia se interesa en la seguridad del paciente. En los aspectos a mejorar se destaca la percepción de personal insuficiente y el alto flujo de personal ocasional percibidos como riesgos en la atención que afecta la seguridad del paciente. Los participantes del estudio indican que al notificar eventos adversos se llevarán a cabo acciones punitivas en su contra. Conclusiones: Las instituciones prestadoras de servicios de salud y su personal son cada vez más conscientes de la importancia de mejorar la seguridad del paciente.
\end{abstract}

Palabras clave: Seguridad del paciente, personal de enfermería, administración de la seguridad.

\footnotetext{
ABSTRACT

The patient safety culture includes the beliefs and attitudes arising from the practice to ensure that no unnecessary harm or potential harm will be experienced associated with healthcare. This study describes the perception of the culture of nursing staff in third level institutions in Bogotá. Design: Descriptive, transversal and exploratory

* Enfermera, profesora Facultad de Enfermería, Universidad Nacional de Colombia, Bogotá, Colombia. Email: olguitago@ gmail.com

${ }_{* *}^{*}$ Enfermera, Universidad Nacional de Colombia, Bogotá, Colombia. Email: waarenasg@unal.edu.co

*** Enfermera, Universidad Nacional de Colombia, Bogotá, Colombia. Email: adr991@yahoo.es

${ }^{* * * *}$ Enfermera, Universidad Nacional de Colombia, Bogotá, Colombia. Email: jgs8901@yahoo.es

${ }^{* * * * *}$ Enfermera, Universidad Nacional de Colombia, Bogotá, Colombia. Email: erikamateus19@yahoo.com

${ }^{* * * * *}$ Enfermera, coordinadora de Enfermería E.S.E. Hospital Simón Bolívar. Bogotá, Colombia, Email: amparosotogamez@ hotmail.com
} 
study performed during the first half of 2009. The sample consists of 201 nursing professionals and technicians who agreed to participate and met the inclusion requirements. The measurement instrument was made up with the Hospital Survey on Patient Safety Culture. The questionnaire was self-administered, after informing the purpose and ethical parameters. Data analysis was carried out using SPSS (Statistical Package for the Social Sciences). Results: The culture of patient safety as reported by the nursing staff is evidenced by positive results in the coordination and effective communication within the units and the general perception that management is interested in patient safety. As for areas to improve, the perception of inadequate staffing and high flow perceived as casual staff in health risks affecting patient safety are highlighted. Participants in the study indicate that in reporting adverse events, punitive actions will take place against them. Conclusions: The institutions providing health services and staff are increasingly aware about the importance of improving patient safety.

Key words: Patient safety, nursing staff, safety management.

Fecha recepción: 09/09/10 Fecha aceptación: 04/10/11

\section{INTRODUCCIÓN}

La preocupación por la seguridad del paciente surge a partir de la documentación de la incidencia de eventos adversos que impactan negativamente en la calidad de la atención en salud, tal como se indicó en la 55a Asamblea Mundial de la Salud en 2002 (1), en la que se mostró el alto costo de estos eventos en términos materiales y de oportunidad para los servicios de salud, y más importante aún, como determinante en la vigilancia y mantenimiento del bienestar del paciente. Esta temática ha sido abordada desde finales del siglo pasado, pero sólo a partir del año 2000, con la publicación del libro "Errar es Humano", del Instituto de Medicina de la Academia Nacional de Ciencias de los Estados Unidos de América, se dimensionó el impacto de los errores en los procesos de atención en salud. Según la Organización Mundial de la Salud (OMS), en los países en vía de desarrollo, el $77 \%$ de los eventos adversos corresponden a casos de medicamentos adulterados y de baja calidad, el mal estado de la infraestructura y de los equipos médicos, el escaso control de las infecciones y la falta de cultura hacia la seguridad del paciente, entre otros, conducen a una mayor probabilidad de ocurrencia de eventos adversos evitables (2).

Por lo tanto, los profesionales de la salud están llamados a hacerse parte, a nivel personal e institucional, de las iniciativas que buscan estudiar y disminuir el impacto negativo de esta problemática en los sistemas de Salud. La OMS, respondiendo a esta problemática, ha propuesto lineamientos que se exponen a través de la Alianza Mundial para la Seguridad del Paciente (3).

Singer et al. (4) y Hellings et al. (5) mencionan la necesidad de implementar estrategias de educación en el ámbito de la seguridad del paciente y específicamente en el reporte oportuno de eventos adversos. En otro referente teórico "Estado del arte del seguimiento a eventos adversos" (6) se evidencian que los eventos adversos no son reportados y se menciona que "el 33\% de los encuestados relatan que cuando existe una equivocación y ésta no afecta al paciente éste no es reportado"; en otro estudio (7) se reporta que los profesionales de la salud, siendo las personas más importantes en el proceso, no reportan oportunamente, por miedo a las acciones punitivas en su contra.

Otro estudio realizado con enfermeras y psicólogos refleja que el 95.2\% de los Enfermeros sugieren considerar la seguridad del paciente actualmente como una cuestión muy importante en la asistencia médica y un componente necesario del cuidado de calidad $(7,8)$.

Sin embargo, a pesar de la relevancia y el 
auge de la temática abordada, relativamente pocos investigadores han examinado el concepto "cultura de seguridad" en la atención en salud, específicamente en relación con el cuidado de enfermería. Esta investigación propone describir la cultura de seguridad del paciente referida por el personal de enfermería en instituciones de tercer nivel en Bogotá.

\section{Marco teórico}

A continuación se presentan los conceptos fundamentales tenidos en cuenta en el trabajo de investigación:

Cultura de seguridad: Corresponde a un grupo de creencias y actitudes que surgen a partir del desarrollo investigativo y la práctica diaria, encaminadas a proporcionar un máximo de bienestar al sujeto de cuidado. Este término abarca tanto el conocimiento como la puesta en marcha de iniciativas que respondan a los lineamientos de seguridad del paciente vigentes en Colombia, contenidos en el Sistema Obligatorio de Garantía de Calidad en Salud (SOGCS). Según el Ministerio de la Protección Social de Colombia, constituye el ambiente de despliegue de las acciones de seguridad del paciente, los cuales deben darse en un entorno de confidencialidad y de confianza entre ellos y los profesionales y la comunidad. Para el sistema Nacional de Salud del Reino Unido (10), este despliegue parte de "la conciencia de que las cosas pueden ir mal," y el reconocimiento de que los errores en la prestación del servicio no están sólo ligados a la persona, sino también al sistema en que ella trabaja. Desde esta óptica, la "cultura de seguridad del paciente”, además de implicar la capacidad de reconocer los errores, involucra la conciencia de que éstos deben ser reportados para que el análisis posterior redunde en mejorar las cosas.

Personal de enfermería: Según el concepto de la Asociación Española de enfermería en Cardiología, son los funcionarios que laboran en o para una organización que proporciona cuidados de enfermería en instituciones $u$ organismos basados en conocimientos científicos. Para este estudio este término incluye a los profesionales y auxiliares o técnicos de enfermería que laboran en las instituciones en las cuales se aplicó el instrumento (11).

Según la Ley 266 de 1996 (12) la enfermería es una profesión liberal y una disciplina de carácter social, cuyos sujetos de atención son la persona, la familia y la comunidad, con sus características socioculturales, sus necesidades y derechos, así como el ambiente físico y social que influye en la salud y en el bienestar.

Instituciones de III nivel: De acuerdo a la Ley 100 de 1993 "las Instituciones Prestadoras de Salud son entidades oficiales, mixtas, privadas, comunitarias y solidarias, organizadas para la prestación de los servicios de salud a los afiliados del Sistema General de Seguridad Social en Salud, dentro de las Entidades Promotoras de Salud o fuera de ellas". Los hospitales de tercer nivel de complejidad en los que se lleva a cabo este proyecto son instituciones prestadoras de salud que están constituidas por recursos humanos, infraestructura y tecnología destinada al diagnóstico y tratamiento de las afecciones que requieran servicios médicos de alta complejidad y especialización (13).

\section{Objetivo general}

Caracterizar la cultura de seguridad del paciente referida por el personal de enfermería en instituciones de tercer nivel en Bogotá, durante el primer semestre del 2009.

\section{Objetivos específicos}

- Explorar la percepción del personal de enfermería de la cultura de seguridad 
del paciente, durante el primer semestre del 2009, mediante el instrumento, versión española, Hospital Survey on Patient Safety Culture.

- Describir la cultura de seguridad del paciente, en las dimensiones descritas por el instrumento versión española, Hospital Survey on Patient Safety Culture: condiciones del servicio o unidad, condiciones del hospital, comunicación en el servicio e información específica acerca de actividades en la institución.

\section{MATERIAL Y MÉTODO}

Para responder el propósito de la investigación se realizó un estudio no experimental de tipo descriptivo exploratorio, de corte transversal, con modalidad de encuesta, realizado entre enero y junio de 2009. La muestra fue tomada de manera aleatoria estratificada, conformada por 201 participantes (110 profesionales y 91 auxiliares de enfermería) que laboraban en instituciones prestadoras de servicios de salud de III nivel de atención que cumplieron los siguientes criterios de inclusión: ser trabajador activo en la institución seleccionada, ser profesional o técnico de enfermería y trabajar en el área clínica asistencial.

Se obtuvo un $\mathrm{N}$ de $411 \mathrm{Zc}=1.96$ ( $\mathrm{a}=$ 0.05 ) y una $\mathrm{k}$ de 0.1 (error de aproximación de la media poblacional en función de la desviación estándar para fines del estudio definitivo) para un resultado de 201 profesionales y auxiliares de enfermería que laboraban en instituciones prestadoras de servicios de salud de III nivel de atención que cumplieron los criterios de inclusión.

$$
n=\frac{N Z_{c}^{2}}{k^{2}(N-1)+Z_{c}^{2}}=\frac{411 \times 1.96^{2}}{0.1^{2}(459-1)+1.96^{2}}=201
$$

Se utilizó la versión española del instrumento Hospital Survey on Patient Safety
Culture (14) adaptado por el grupo de investigación en gestión de calidad de la Universidad de Murcia (15), concebido como una herramienta para examinar de manera fiable las conductas y actitudes de los trabajadores del área de la salud relacionadas con la seguridad del paciente, y permite obtener información acerca de la cultura de seguridad y posteriormente identificar fortalezas y debilidades. Se trata de un cuestionario auto-administrado. La valoración de cada ítem se realiza por medio de la escala de Likert, en 5 grados de acuerdo a cada sección, para las secciones A y B: 1 muy en desacuerdo; 2 en desacuerdo; 3 indiferente; 4 de acuerdo; 5 muy de acuerdo, y para las secciones $C$ y D: 1 nunca; 2 casi nunca; 3 a veces; 4 casi siempre; 5 siempre. Inicialmente agrupa las preguntas bajo cuatro criterios: "Su Servicio/Unidad", "su hospital", "comunicación en su servicio/unidad" e "información complementaria". Las preguntas del cuestionario original se clasifican en 12 dimensiones, cuyo significado se describe en la Tabla $1(12,16)$. Los datos estadísticos fueron analizados con un método descriptivo exploratorio univariado usando el programa SPSS versión 15 y los resultados significativos $(\mathrm{p}<0.05)$ se expresaron como medidas de tendencia central y medidas de dispersión, tomadas de forma independiente para cada una de las secciones A, B, y C del instrumento. Los resultados de la sección $\mathrm{D}$ se presentan con medidas de frecuencias y porcentajes, a razón de que esta sección contiene la información complementaria acerca de los participantes. Para la aplicación y validación de la versión española del instrumento se realizó una prueba piloto para garantizar la calidad del instrumento en términos de validez facial entre 36 participantes, encontrando que los ítemes de la encuesta cumplieron con los criterios de claridad, precisión y comprensión. Así mismo se realizaron pruebas de confiabilidad de cada una de las dimensiones en la versión en español, calculando 
el alfa de Cronbach, que se presenta en la Tabla 1, encontrando que en todos los casos la fiabilidad es de aceptable a excelente.

\section{RESULTADOS}

\section{Sección A: Su servicio / unidad}

La media general que obtuvo la sección A fue de 3,31. La media mínima por preguntas pertenece a las preguntas A22 (Mi superior) jefe pasa por alto los problemas de seguridad del paciente que ocurren habitualmente) y A2 (Hay suficiente personal para afrontar la carga de trabajo) con una media de 2.16 cada una. La media máxima pertenece a la pregunta $\mathrm{A} 4$ (En esta unidad nos tratamos todos con respeto) con 4.03.

La desviación típica general para la sección A es de 1.21, la desviación mínima por preguntas de la sección A pertenece a la pregunta A9 (Cuando se detecta algún fallo en la atención al paciente se llevan a cabo las medidas apropiadas para evitar que ocurra de nuevo), con 0.96 la desviación máxima pertenece a la pregunta $\mathrm{A} 10$ (No se producen más fallos por casualidad) con 1.57. En cuanto a la asimetría general para la sección A arrojó una asimetría negativa con una desviación superior a la izquierda de -0.74 . La curtosis general en la sección A fue de 0.82 con una concentración Leptocúrtica.

\section{Sección B: Su hospital}

La media general fue de 3.01. La media mínima fue 2.56 en la pregunta B33 (Surgen problemas en la atención de los pacientes como consecuencia de los cambios de turno). La media máxima por pregunta fue de 3.46 en la pregunta B32 (Los servicios/unidades trabajan de forma coordinada entre sí para proporcionar la mejor atención posible a los pacientes.), siendo esta misma pregunta la que presentó la mínima desviación estándar con
1.15. La pregunta con máxima desviación estándar fue la B30 (La gerencia o dirección del hospital muestra con hechos que la seguridad del paciente es una de sus prioridades) con 1.38. La asimetría general fue negativa con desviación hacia la izquierda de -0,29. La curtosis general para la sección B muestra un puntaje de -0.69 que corresponde a una distribución de datos platicúrtica.

\section{Sección C: Comunicación en su servicio/ unidad}

La media general que obtuvo la sección $\mathrm{C}$ fue de 3.23. La media mínima fue de 2.47 para la pregunta C39 que indaga acerca del temor del personal a hacer preguntas sobre lo que parece que se había hecho de forma incorrecta. Las preguntas con el resultado de media máxima son la C36 (Se nos informa de los errores que ocurren en este servicio/ unidad) y la C40 (Se notifican los errores que son descubiertos y corregidos antes de afectar al paciente) con 3.72. La asimetría general fue negativa con desviación hacia la izquierda de $-0,67$. La curtosis general en la sección $C$ es de 0.47 con una concentración mesocúrtica.

Al consolidar la calificación media obtenida en las secciones anteriores se encuentra que estas secciones son en general percibidas de forma positiva por los participantes:

\section{Sección D: Información complementaria}

En la sección D se evidencia un patrón en el cual los mayores porcentajes de trabajadores de salud encuestados afirman haber empezado a trabajar en el lapso 2001-2009 (36\%) y en el mismo lapso de tiempo haber empezado a desempeñarse en el hospital y servicio que trabajan actualmente $(42.7 \%$ y $64.7 \%$ de la muestra, respectivamente). Así mismo un $89 \%$ (179 individuos) aseguraron mantener contacto directo con el paciente en su trabajo habitual como enfermeros profesionales $(32.3 \%)$ o auxiliares de enfermería (50.2\%); al indagar respecto a los 
incidentes reportados el último año, 128 individuos (63.6\% de la muestra) afirmaron que no habian reportado ningún incidente, y 68 individuos (33.8\%) afirmaron que habían reportado menos de 10 incidentes. En el análisis de las preguntas relacionadas con el proceso de elaboración de historias clínicas se encuentra que el $48 \%$ de los participantes afirma que son informados los cambios de medicación de los pacientes de forma clara y rápida, 35.8 \% afirma que antes de realizar una nueva prescripción de medicamentos se tiene en cuenta los medicamentos que está tomando el paciente, y apenas un 28\% (58 individuos) indica que cualquier información que afecte al diagnóstico del paciente es comunicada de forma clara y rápida a todos los profesionales implicados en la atención de ese paciente.

El análisis de la pregunta adicional ¿Considera importante que en la institución se establezcan estándares (Modelo/teoría) de cuidado seguro para la asistencia? ¿Podría referenciar alguno? evidencia que las teorías de enfermería, si bien son ampliamente divulgadas en la formación profesional, no son aplicadas de manera práctica en el quehacer diario de los enfermeros profesionales, pues aunque el $40.7 \%$ afirmó que sería importante establecer el estándar, apenas 5 participantes pudieron referenciar una teoría de enfermería concreta.

\section{DISCUSIÓN Y COMENTARIO}

Entendiendo la cultura de seguridad del paciente como la suma del conocimiento y las actitudes cotidianas del equipo de enfermería que tienden a evitar, disminuir o mitigar el daño potencial evitable durante la atención en salud, este estudio caracterizo la cultura de seguridad del paciente referida por el personal de enfermería en instituciones de III nivel en Bogotá, durante el primer se- mestre del 2009, se identificaron algunos de los aspectos más favorables y problemáticos, que forman parte fundamental de esta cultura de seguridad.

El análisis producto de la sección A (su servicio/unidad) reveló de manera positiva que el que el superior o jefe no pasa por alto los problemas de seguridad del paciente, sino que por el contrario son tomadas medidas correctivas, como Refiere el Ministerio de Sanidad y Consumo Español y la Universidad de Murcia (15). En esta misma sección sobresale la percepción de que el personal que labora en las diferentes unidades no es el suficiente para garantizar la prestación de los servicios de manera óptima, lo cual se traduce en aumento de la carga laboral e impacta negativamente en la percepción del personal en cuanto al ambiente propicio para desarrollar conductas que favorezcan la cultura de seguridad; esta situación la expuso Singer et al. (4) al afirmar que "la cultura de la seguridad no puede ser tan fuerte como se desea" ya que la percepción acerca de la misma varía de persona a persona, y según las condiciones de trabajo y la carga laboral; este hecho apoya los resultados de diferentes estudios previos $(16,17,18)$ donde la mayoría de la población encuestada opinó que en el servicio no cuentan con el suficiente personal para manejar la carga del trabajo, lo que a su vez atenta contra el principio de oportunidad $(19,20)$. Los profesionales y técnicos en enfermería están llamados a generar y exigir acciones que mejoren las dinámicas de trabajo de las unidades hospitalarias para asegurar un cuidado de calidad (21).

A su vez los resultados de la sección B (su hospital) muestran que los participantes contribuyen a la cultura de seguridad del paciente desde su área específica de trabajo, sintiéndose más seguros al actuar específicamente en ella $(22,23,24)$. Un estudio que midió la cultura de seguridad del paciente en cinco hospitales británicos (25) encontró 
una cuestión de mayor complejidad al respecto ya que los resultados demostraron que cuando la seguridad es una prioridad débil, hay más errores que cuando la gestión de las prácticas de seguridad es importante en el hospital como organización.

Por otra parte el análisis de los datos de la sección C (comunicación en su servicio/unidad) muestran que la notificación de eventos adversos y la toma de decisiones en equipo son aspectos que el personal considera de gran importancia, así como la confianza dentro del equipo de salud, sin embargo resalta que el reporte de eventos sólo se realiza cuando se ha afectado de alguna forma al paciente y es éste el primero en informarlo, esto ratifica lo evidenciado por Murillo (6) (en el cual fue utilizado el mismo instrumento de evaluación), quien afirma que "el 33\% de los encuestados relatan que cuando existe una equivocación y ésta no afecta al paciente éste no es reportado", "Los sistemas de salud deben pasar de la "culpa y la vergüenza" cultura que impide el reconocimiento del error $y$, por tanto, obstaculiza cualquier posibilidad de aprender de éstos, a una en la que se tenga fácil acceso a la información que apoye el aprendizaje de la experiencia con el fin de promover los sistemas de prevención de errores $(26,27)$.

Meyer (28) asegura que la confianza y el clima organizacional en el reporte y mejora de los eventos influyen en la seguridad del paciente, pues "cuando hay miedo en el hospital, el personal hará todo lo posible para ocultar los errores y el filtro de datos" (24, 28). Por lo tanto es necesario implementar en los servicios de salud diferentes estrategias que permitan comprometer a los trabajadores profesionales y técnicos con el reporte, la necesidad de actualización en los conocimientos para la atención del paciente, según lo señalado por Maddox et al. (9) que proponen cambios en los métodos de educación de enfermería creando un ambiente de aprendizaje, con fluida comunicación abierta, que permita a las enfermeras identificar, discutir $y$, en última instancia, evitar los errores en la atención de la salud $(29,30)$.

El análisis de la sección D mostró un patrón en el cual los mayores porcentajes de la muestra afirman haber empezado a trabajar en el lapso 2001-2009 y en el mismo lapso de tiempo haber empezado a desempeñarse en el hospital y servicio que trabajan actualmente, sin embargo resulta paradójico que a pesar de que la mayoría de los participantes en el estudio afirmaron tener contacto directo con el paciente, al indagar respecto a los incidentes reportados el último año, un gran porcentaje de ellos afirmó que no había reportado ningún incidente, y tan sólo una tercera parte de la muestra manifestó haber reportado menos de 10 incidentes; esto puede ser un indicio de las debilidades en la cultura del reporte de incidentes y eventos adversos pues, a pesar de que se está en contacto directo con el sujeto de cuidado, no se reportan (en su mayoría) los eventos adversos en el cuidado cotidiano, lo que se puede deber a la percepción que tienen estos encuestados respecto de las acciones punitivas que puede llevar a cabo. Es así como diversos autores resaltan la importancia de la respuesta no punitiva al error ya que en dicho estudio el $35.5 \%$ de los encuestados relatan tener preocupación que al reportar los errores se guarden en su hoja de vida y el $29.1 \%$ de los encuestados opinan que al reportar sus errores, se vuelve en contra de ellos $(31,32)$.

Meyer (28) relacionó la falta de información-comunicación por parte del personal sanitario con una mayor preocupación de sufrir un error en la atención. Este aporte permite visualizar que la información realmente es la base de la implementación de una cultura de seguridad del paciente (33, 34).

En la discusión de la pregunta adicional ¿Considera importante que en la institución se establezcan estándares (modelo/teoría) de 
cuidado seguro para la asistencia? ¿Podría referenciar alguno? Se destaca que a pesar de la existencia de trabajos en la literatura acerca de modelos y teorías de enfermería, se evidencia en los participantes que existe un gran vacío entre la teoría y la práctica (35, 36) y sin lugar a dudas, esta brecha debe ser acortada, pues "las teorías y modelos de enfermería cumplen un rol importantísimo, ya que proporcionan una base para formular hipótesis relativas a la práctica, que hacen posible obtener un fundamento para las acciones de enfermería" (37). Para el desarrollo futuro de la profesión será indispensable que cada Departamento de Enfermería y cada individuo se empodere de un paradigma del Cuidado, cosa que enriquecerá no sólo el sentido de pertenencia hacia la disciplina, sino que redundará en mayor calidad y compromiso en la atención a los pacientes $(38,39)$.

Autores señalan que las directrices no toman en cuenta suficientemente las preferencias de los pacientes. Las preferencias, en su sentido más amplio representan la conveniencia de un resultado relacionado con la salud, el proceso, o la opción de tratamiento $(40,41)$. En otros trabajos de investigación, al examinar si durante la atención clínica se indagan de forma anticipada las preferencias del paciente sobre tratamientos y procedimientos de soporte vital, la respuesta predominante que correspondió a un 14,4\% de los encuestados afirman hacerlo sólo 'a veces'. Por lo tanto una opción de mejora sería seguir implementando las guías de práctica clínica, ya que como enuncian $(42,43)$, las guías de práctica clínica son declaraciones sistemáticamente desarrolladas para ayudar a tomar decisiones dentro del equipo de salud como las de los pacientes, con el objetivo de mejorar la calidad de cuidado, manejando conceptos como las preocupaciones, intereses, deseos, y expectativas de éstos, todo lo cual forma parte indispensable de la cultura de seguridad del paciente $(43,44)$.

\section{CONCLUSIONES}

- La caracterización de la cultura de seguridad del paciente referida por el personal de enfermería, en instituciones de tercer nivel en Bogotá, permitió evidenciar los valores y creencias de un recurso humano valioso e indispensable en el cuidado de la salud de las personas, por medio de la percepción de dicho personal acerca de las diferentes dimensiones sugeridas por el instrumento Hospital Survey on $\mathrm{Pa}$ tient Safety Culture Versión Española, lo que genera un importante aporte para el abordaje de las intervenciones institucionales en pro del mejoramiento de la calidad de la atención en salud.

- Los siguientes aspectos que hacen parte de la cultura de seguridad y se destacan como relevantes en la percepción de los participantes: la comunicación oportuna, efectiva y de calidad dentro de los miembros del equipo de salud para la identificación de oportunidades de mejora en la correcta atención del paciente. Así mismo la retroalimentación de los errores en la atención y de sus respectivas alternativas de solución, además de prevenir futuros eventos adversos en el cuidado del paciente, permite entablar una relación de confianza y compromiso dentro del equipo de salud encargado del mismo.

- Los hallazgos actuales y previos en cultura de seguridad del paciente constituyen un aporte disciplinar importante, pues primero documentan y, luego, respaldan las acciones que en este campo se llevan a cabo en la práctica clínica, y además son el primer paso hacia la construcción unificada de paramentos de actuación ante situaciones específicas de la práctica de enfermería respecto de la seguridad del paciente, lo que permite el fortalecimiento de los equipos de enfermería a modo individual e interdisciplinar, y sin duda se pone de relieve en la calidad y seguridad de la atención. 


\section{CONFLICTO DE INTERÉS}

\section{Los autores declaran no tener conflictos de interés.}

\section{Tabla 1. Dimensiones (secciones de la cultura de seguridad y los ítemes que incluyen).}

\begin{tabular}{|c|c|}
\hline \multicolumn{2}{|c|}{ A. RESULTADOS DE LA CULTURA DE SEGURIDAD } \\
\hline 1. Frecuencia de eventos notificados. & $\begin{array}{l}\text { - Se notifican los errores que son descubiertos y corregidos antes de afectar al } \\
\text { paciente (P 40). } \\
\text { - Se notifican los errores que previsiblemente no van a dañar al paciente (P. 41). } \\
\text { - Se notifican los errores que no han tenido consecuencias adversas, aunque } \\
\text { previsiblemente podrían haber dañado al paciente (P 42). }\end{array}$ \\
\hline 2. Percepción de seguridad & $\begin{array}{l}\text { - Nunca se aumenta el ritmo de trabajo si eso implica sacrificar la seguridad del } \\
\text { paciente (P 15). } \\
\text { - Nuestros procedimientos y medios de trabajo son buenos para evitar errores } \\
\text { en la asistencia (P. 18). } \\
\text { - No se producen más fallos por casualidad (P 10). } \\
\text { - En esta unidad hay problemas relacionados con la "Seguridad del paciente" } \\
\text { (P 17). }\end{array}$ \\
\hline \multicolumn{2}{|c|}{ B. DIMENSIONES DE LA CULTURA DE SEGURIDAD A NIVEL DE UNIDAD/SERVICIO } \\
\hline $\begin{array}{l}\text { 3. Expectativas y acciones de la } \\
\text { dirección/ supervisión de la } \\
\text { Unidad/Servicio que favorecen la } \\
\text { Seguridad }\end{array}$ & $\begin{array}{l}\text { - Mi superior/jefe expresa su satisfacción cuando intentamos evitar riesgos en la } \\
\text { seguridad del paciente (P 19). } \\
\text { - Mi superior/jefe tiene en cuenta, seriamente, las sugerencias que le hace el } \\
\text { personal para mejorar la seguridad del paciente (P 20). } \\
\text { - Cuando aumenta la presión del trabajo, mi superior/jefe pretende que } \\
\text { trabajemos más rápido, aunque se pueda poner en riesgo la seguridad del } \\
\text { paciente (P 21). } \\
\text { - Mi superior/jefe pasa por alto los problemas de seguridad del paciente que } \\
\text { ocurren habitualmente (P 22). } \\
\text { - Tenemos actividades dirigidas a mejorar la seguridad del paciente (P 6). } \\
\text { - Cuando se detecta algún fallo en la atención al paciente se llevan a cabo las } \\
\text { medidas apropiadas para evitar que ocurra de nuevo (P 9). } \\
\text { - Los cambios que hacemos para mejorar la seguridad del paciente se evalúan } \\
\text { para comprobar su efectividad (P 13). }\end{array}$ \\
\hline $\begin{array}{l}\text { 5. Trabajo en equipo en la } \\
\text { Unidad/Servicio }\end{array}$ & $\begin{array}{l}\text { - El personal se apoya mutuamente (P 1). } \\
\text { - Cuando tenemos mucho trabajo, colaboramos todos como un equipo para } \\
\text { poder terminarlo (P 3). } \\
\text { - En esta unidad nos tratamos todos con respeto (P 4). } \\
\text { - Cuando alguien está sobrecargado de trabajo, suele encontrar ayuda en los } \\
\text { compañeros (P 11). }\end{array}$ \\
\hline 6. Franqueza en la comunicación & $\begin{array}{l}\text { - Cuando el personal ve algo que puede afectar negativamente a la atención que } \\
\text { recibe el paciente, habla de ello con total libertad (P 35). } \\
\text { - El personal puede cuestionar con total libertad las decisiones o acciones de sus } \\
\text { superiores (P 37). } \\
\text { - El personal teme hacer preguntas sobre lo que parece que se ha hecho de forma } \\
\text { incorrecta (P 39). }\end{array}$ \\
\hline
\end{tabular}


Continuación Tabla 1.

\begin{tabular}{|c|c|}
\hline 7. Feed-back y comunicación sobre errores & $\begin{array}{l}\text { - Cuando notificamos algún incidente, nos informan sobre qué tipo de } \\
\text { actuaciones se han llevado a cabo (P 34). } \\
\text { - Se nos informa de los errores que ocurren en este servicio / unidad (P 36). } \\
\text { - En mi servicio / unidad discutimos de qué manera se puede evitar que un error } \\
\text { vuelva a ocurrir (P 38). }\end{array}$ \\
\hline 8. Respuesta no punitiva a los errores & $\begin{array}{l}\text { - Si los compañeros o los superiores se enteran de que has cometido algún error, } \\
\text { lo utilizan en tu contra (P 8). } \\
\text { - Cuando se detecta algún fallo, antes de buscar la causa, buscan un "culpable" } \\
\text { (P 12). } \\
\text { - Cuando se comete un error, el personal teme que eso quede en su expediente } \\
\text { (P 16). }\end{array}$ \\
\hline 9. Dotación de personal & $\begin{array}{l}\text { - Hay suficiente personal para afrontar la carga de trabajo (P 2). } \\
\text { - A veces no se puede proporcionar la mejor atención al paciente porque la } \\
\text { jornada laboral es agotadora (P 5). } \\
\text { - En ocasiones no se presta la mejor atención al paciente porque hay demasiados } \\
\text { sustitutos o personal temporal (P7). } \\
\text { - Trabajamos bajo presión para realizar demasiadas cosas demasiado deprisa } \\
(\mathrm{P} 14) \text {. }\end{array}$ \\
\hline $\begin{array}{l}\text { 10. Apoyo de la gerencia del hospital en la } \\
\text { seguridad del paciente }\end{array}$ & $\begin{array}{l}\text { - La gerencia o la dirección del hospital facilita un clima laboral que favorece la } \\
\text { seguridad del paciente (P 23). } \\
\text { - La gerencia o la dirección del hospital muestra con hechos que la seguridad del } \\
\text { paciente es una de sus prioridades (P 30). } \\
\text { - La gerencia / dirección del hospital sólo parece interesarse por la seguridad } \\
\text { del paciente cuando ya ha ocurrido algún suceso adverso en un paciente (P 31). }\end{array}$ \\
\hline \multicolumn{2}{|c|}{ C. DIMENSIONES DE LA CULTURA DE SEGURIDAD A NIVEL DE TODO EL HOSPITAL } \\
\hline 11. Trabajo en equipo entre unidades/servicios & $\begin{array}{l}\text { - Hay una buena cooperación entre las unidades / servicios que tienen que } \\
\text { trabajar conjuntamente (P 26). } \\
\text { - Los servicios/unidades trabajan de forma coordinada entre sí para } \\
\text { proporcionar la mejor atención posible (P 32). } \\
\text { - Las diferentes unidades del hospital no se coordinan bien entre ellas (P 24). } \\
\text { - Suele resultar incómodo tener que trabajar con personal de otros servicios/ } \\
\text { unidades (P 28). }\end{array}$ \\
\hline $\begin{array}{l}\text { 12. Problemas en cambios de turno y } \\
\text { transiciones entre servicios/unidades }\end{array}$ & $\begin{array}{l}\text { - La información de los paciente se pierde, en parte, cuando éstos se transfieren } \\
\text { desde una unidad /servicio a otra (P 25). } \\
\text { - En los cambios de turno se pierde con frecuencia información importante } \\
\text { sobre la atención que ha recibido el paciente (P 27). } \\
\text { - El intercambio de información entre los diferentes servicios es habitualmente } \\
\text { problemático (P 29). } \\
\text { - Surgen problemas en la atención de los pacientes como consecuencia de los } \\
\text { cambios de turno (P 33). }\end{array}$ \\
\hline
\end{tabular}


Tabla 2. Fiabilidad de las dimensiones contenidas en el cuestionario.

\begin{tabular}{|l|c|}
\hline Dimensión (secciones) & Alfa de Cronbach \\
\hline Frecuencia de eventos notificados & 0.88 \\
\hline Percepción de seguridad & 0.65 \\
\hline $\begin{array}{l}\text { Expectativas y acciones de la dirección/ } \\
\text { supervisión de la Unidad/Servicio que } \\
\text { favorece la seguridad }\end{array}$ & 0.84 \\
\hline $\begin{array}{l}\text { Aprendizaje organizacional/mejora } \\
\text { continua }\end{array}$ & 0.68 \\
\hline Trabajo en equipo en la Unidad/Servicio & 0.82 \\
\hline Franqueza en la comunicación & 0.66 \\
\hline Feed-back y comunicación sobre errores & 0.73 \\
\hline Respuesta no punitiva a los errores & 0.65 \\
\hline Dotación de personal & 0.64 \\
\hline $\begin{array}{l}\text { Apoyo de la gerencia del hospital en la } \\
\text { seguridad del paciente }\end{array}$ & 0.81 \\
\hline Trabajo en equipo entre unidades & 0.73 \\
\hline $\begin{array}{l}\text { Problemas en cambios de turno y } \\
\text { transiciones entre servicios/unidades }\end{array}$ & 0.74 \\
\hline
\end{tabular}

Tabla 3. Análisis estadístico sección A: su servicio/ unidad.

\begin{tabular}{|c|c|c|c|c|c|c|}
\hline SECCIÓN & MÍNIMO & MÁXIMO & MEDIA & DESV. TIP. & ASIMETRÍA & CURTOSIS \\
\hline Al & 0 & 5 & 3,64 & 1,36 & $-1,64$ & 1,86 \\
\hline $\mathrm{A} 2$ & 0 & 5 & 2,16 & 1,16 & 0,63 & $-0,15$ \\
\hline $\mathrm{A} 3$ & 0 & 5 & 3,68 & 1,1 & $-1,24$ & 1,6 \\
\hline A4 & 0 & 5 & 4,03 & 0,97 & $-1,53$ & 3,03 \\
\hline A5 & 0 & 5 & 3,73 & 1,11 & $-1,046$ & 0,59 \\
\hline A6 & 0 & 5 & 3,74 & 1,12 & $-1,73$ & 3,1 \\
\hline A7 & 0 & 5 & 3 & 1,26 & $-0,045$ & $-1,2$ \\
\hline A8 & 0 & 5 & 2,83 & 1,29 & $-0,14$ & -102 \\
\hline A9 & 0 & 5 & 4,01 & 0,96 & $-2,02$ & 5,23 \\
\hline A10 & 0 & 5 & 2,87 & 1,57 & $-0,45$ & $-0,8$ \\
\hline A11 & 0 & 5 & 3,7 & 1 & $-0,99$ & 0,8 \\
\hline A12 & 0 & 5 & 3,33 & 1,36 & $-0,59$ & $-0,67$ \\
\hline $\mathrm{A} 13$ & 0 & 5 & 3,6 & 1,17 & $-0,17$ & 1,01 \\
\hline A14 & 0 & 5 & 3,34 & 1,35 & $-0,58$ & $-0,63$ \\
\hline A15 & 0 & 5 & 2,63 & 1,36 & $-0,04$ & $-0,78$ \\
\hline
\end{tabular}


Continuación Tabla 3.

\begin{tabular}{|c|c|c|c|c|c|c|}
\hline A16 & 0 & 5 & 3,9 & 1,12 & $-1,6$ & 2,63 \\
\hline A17 & 0 & 5 & 3,25 & 1,14 & $-0,45$ & $-0,9$ \\
\hline A18 & 0 & 5 & 3,8 & 1,035 & $-1,67$ & 3,35 \\
\hline A19 & 0 & 5 & 3,35 & 1,3 & $-0,73$ & $-0,17$ \\
\hline A20 & 0 & 5 & 3,51 & 1,2 & $-0,14$ & 0,68 \\
\hline A21 & 0 & 5 & 2,65 & 1,25 & $-0,24$ & $-0,8$ \\
\hline A22 & 0 & 5 & 2,16 & 1 & 1,18 & 1,3 \\
\hline $\begin{array}{c}\text { CONSOLIDADO } \\
\text { SECCIÓN A }\end{array}$ & $\mathbf{0}$ & $\mathbf{5}$ & $\mathbf{3 , 3 1}$ & $\mathbf{1 , 2 1}$ & $\mathbf{- 0 , 7 4}$ & $\mathbf{0 , 8 2}$ \\
\hline
\end{tabular}

Tabla 4. Análisis estadístico sección B: Su Hospital.

\begin{tabular}{|l|c|c|c|c|c|c|}
\hline \multicolumn{1}{|c|}{ SECCIÓN } & MÍNIMO & \multicolumn{1}{c}{ MÁXIMO } & \multicolumn{1}{c}{ MEDIA } & \multicolumn{1}{c|}{ DESV. TIP. } & \multicolumn{1}{c|}{ ASIMETRÍA } & \multicolumn{1}{c|}{ CURTOSIS } \\
\hline B23 & 0 & 5 & 3,11 & 1,2 & $-0,43$ & $-0,70$ \\
\hline B24 & 0 & 5 & 3,12 & 1,3 & $-0,44$ & $-0,72$ \\
\hline B25 & 0 & 5 & 2,98 & 1,2 & $-0,2$ & $-1,08$ \\
\hline B26 & 0 & 5 & 3,19 & 1,17 & $-0,52$ & $-0,5$ \\
\hline B27 & 0 & 5 & 3,13 & 1,21 & $-0,23$ & $-1,22$ \\
\hline B28 & 0 & 5 & 2,75 & 1,19 & 0,2 & $-0,63$ \\
\hline B29 & 0 & 5 & 2,78 & 1,27 & $-0,19$ & $-0,53$ \\
\hline B30 & 0 & 5 & 3,28 & 1,38 & $-0,66$ & $-0,68$ \\
\hline B31 & 0 & 5 & 2,77 & 1,37 & 0,19 & $-1,01$ \\
\hline B32 & 0 & 5 & 3,46 & 1,15 & $-0,95$ & 0,27 \\
\hline B33 & 0 & 5 & 2,56 & 1,29 & 0,17 & $-0,82$ \\
\hline $\begin{array}{c}\text { CONSOLIDADO } \\
\text { SECCIÓN B }\end{array}$ & $\mathbf{0}$ & $\mathbf{5}$ & $\mathbf{3 , 0 1}$ & $\mathbf{1 , 2 7}$ & $\mathbf{- 0 , 2 9}$ & $\mathbf{- 0 , 6 8}$ \\
\hline
\end{tabular}

Tabla 5. Análisis estadístico sección C: comunicación en su servicio/ unidad.

\begin{tabular}{|c|c|c|c|c|c|c|}
\hline SECCIÓN & MÍNIMO & MÁXIMO & MEDIA & DESV. TIP. & ASIMETRÍA & CURTOSIS \\
\hline C34 & 0 & 5 & 2,96 & 1,24 & $-0,48$ & $-0,14$ \\
\hline C35 & 0 & 5 & 3,35 & 1,17 & $-0,59$ & 0,21 \\
\hline $\mathrm{C} 36$ & 0 & 5 & 3,72 & 1,16 & $-0,93$ & 0,78 \\
\hline C37 & 0 & 5 & 2,32 & 1,12 & 0,24 & $-0,37$ \\
\hline C38 & 0 & 5 & 3,46 & 1,15 & $-0,89$ & 0,89 \\
\hline C39 & 0 & 5 & 2,47 & 1,3 & $-0,36$ & $-0,52$ \\
\hline $\mathrm{C} 40$ & 0 & 5 & 3,72 & 1,09 & $-0,97$ & 1,59 \\
\hline $\mathrm{C} 41$ & 0 & 5 & 3,48 & 1,23 & $-1,05$ & 1,12 \\
\hline $\mathrm{C} 42$ & 0 & 5 & 3,52 & 1,35 & $-1,05$ & 0,72 \\
\hline $\begin{array}{l}\text { CONSOLIDADO } \\
\text { SECCIÓN C }\end{array}$ & 0 & 5 & 3,23 & 1,2 & $-0,67$ & 0,47 \\
\hline
\end{tabular}




\section{REFERENCIAS}

1. Organización Panamericana de la Salud. 55a Asamblea Mundial de la Salud WHA 55.18. Punto 13.9 del orden del día 18 de mayo de 2002. Calidad de la atención: seguridad del paciente. [Internet] [citado 13 febrero 2009]. Disponible http:// www.paho.org/Spanish/AD/THS/EV/ blood-4ta-resolucion.pdf.

2. Fundación Santa Fe de Bogotá. Oficina de Atención Confiable. Seguridad del paciente: implementación de una cultura. [Internet] Bogotá, Colombia. [citado 8 noviembre 2009]. Disponible en: http://www.encolombia.com/medicina/ enfermeria/EnfermeriaVol1042007/Editorial.htm.

3. Organización Mundial de la Salud. Alianza Mundial para la Seguridad del Paciente. [Internet] Ginebra, Suiza [citado el 17 de febrero de 2009]. Disponible en: http://www.who.int/patientsafety/ en/brochure_final

4. Singer S, Gaba D, Geppert J; Sinaiko A, Howard S, Park K. The culture of safety: results of an organization-wide survey in 15 California hospitals. Qual Saf Health Care 2003; 12: 112-118.

5. Hellings J, Schrooten W, Klazinga N, Vleugels A. Challenging patient safety culture: survey results. Int J Health Care Qual Assur International. 2007; 20 (7): 620-632.

6. Murillo D. La gestión de enfermería y los servicios generales en las organizaciones. Cap 5. España: Ediciones Diaz de Santos; 2006.

7. Nieva VF, Sorra, J. Safety culture assessment: a tool for improving patient safety in healthcare organizations. Qual Saf Health Care 2003; 12 (Suppl II): 1723.

8. Vangeest J, Cummins D. An Educational Needs Assessment for Improving Patient Safety Results of a National Study of
Physicians and Nurses. National Patient Safety Foundation ${ }^{\circledR}$. [Internet] Ontario, Canadá [citado el 13 de febrero de 2009] Disponible en http://www.npsf.org/ download/EdNeedsAssess.pdf.

9. Maddox P, Wakefield M, Bull J. Patient safety and the need for professional and educational change. Nurs Outlook. 2001;49 (1): 8-13.

10. Sistema Nacional de Salud (NHS). Agencia Nacional para Seguridad del Paciente (NPSA). Agencia de calidad del sistema nacional de salud. La seguridad del paciente en siete pasos. [Internet] Reino Unido [Citado el 17 de febrero de 2009]. Disponible en: http://www.sefh.es/carpetasecretario/7_PASOS.pdf.

11. Asociación Española de Enfermería en Cardiología. Personal de Enfermería. Enferm Cardiol. 2010;17(50):57-60. [Internet] Madrid, España [citado el 16 de febrero de 2009]. Disponible en: http:// www.enfermeriaencardiologia.com/revista/clavep.htm.

12. Congreso de la República de Colombia. Ley 266 de 1996. Por la cual se reglamenta el ejercicio de la profesión de enfermería en Colombia y se dictan otras disposiciones. Disponible en: http://www. elabedul.net/Documentos/Leyes/1996/ Ley_266.pdf consultado el 20 de septiembre de 2011.

13. Congreso de la Republica de Colombia. Ley 100 de 1993. Por la cual se crea el Sistema General de Seguridad Social Integral y se dictan otras disposiciones. [Internet] Colombia [Citado el 20 de septiembre de 2011]. Disponible en: http://www.colombia.com/actualidad/ images/2008/leyes/ley100.pdf

14. Agency for Health Care Research and Quality. AHRQ. Estados Unidos. [Internet] [Acceso el 24 de febrero de 2009]. Disponible en: http://www.ahrq.gov/.

15. Gobierno de España. Ministerio de Sanidad y Consumo. Madrid. Cuestionario sobres seguridad de los pacientes: 
versión española del Hospital Survey on Patient Safety. [Internet] España [Citado el 24 de febrero de 2009]. Hallado en: http://www.msc.es/organizacion/sns/ planCalidadSNS/docs/CuestionarioSeguridadPacientes1.pdf

16. Reyes L, Jara P, Merino J. Adherencia de las enfermeras/os a utilizar un modelo teórico como base de la valoración de enfermería. Cienc Enferm. 2007; 13 (1): 45-57.

17. Steinberg K. Wellness in every stage of life: a new paradigm for public health programs. Prev Chronic Dis [Internet]. 2007 Jan [citado 10 enero 2009]; 4(1). Disponible en: http://www.cdc.gov/pcd/ issues/2007/jan/06_0120.htm

18. Boutin-Foster C. In spite of good intentions: patients' perspectives on problematic social support interactions. Health Qual Life Outcomes [Internet]. 2005 Sep 5 [citado 7 enero 2009]; 3(1). Disponible en: http://www.hqlo.com/ content/3/1/52

19. Finfgeld-Connett D. Letter to the editor on "Nurse Safety: Have We Addressed the Risks?” Online J Issues Nurs. [Internet]. 2006 Mar. 27 [citado el 10 de enero de 2009];11(2). Disponible en: http://www. nursingworld.org/ojin/letters/t22e6.htm

20. Cipriano Silva M, Ludwick R. Is the doctor of nursing practice ethical? Online J Issues Nurs [Internet]. 2006 Mar. 20 [citado 9 julio 2009]; 11(2). Disponible en: http://nursingworld.org/ojin/ethicol/ ethics_17.htm

21. Meyer G, Foster N, Christrup S, Eisenberg J. Setting a research agenda for medical errors and patient safety. Health Serv Res [Internet]. 2001 Apr. [citado en julio 9 de 2009]; 36 (1 Pt 1). Disponible en: http:// www.pubmedcentral.nih.gov/picrender. fcgi? artid=1089210\&blobtype = pdf

22. Jarousse LA. A vision for value: hospitalpayer partnerships. Hosp Health Netw. 2011; 85(11): 41-8.

23. Piper LE. The ethical leadership cha- llenge: creating a culture of patient- and family-centered care in the hospital setting. Health Care Manag (Frederick). 2011;30(2): 125-32.

24. Bhat YM, McGrath KM, Bielefeldt K. Bravo pH monitoring: should we exclude the first 6 hours? [abstract]. Gastroenterology. 2002;128(4 Suppl 2):392.

25. Vincent C, Neale G, Woshynowych M. Adverse events in British hospitals: preliminary retrospective record review. BMJ. 2001; 322:517-9.

26. Fire safety [disk]. Version 1 for Windows. Oakbrook Terrace (IL): Joint Commission on Accreditation of Healthcare Organizations; c1997. 10 computer disks: color, 3 1/2 in. Accompanied by: 1 manual. Joint publication of the Florida Hospital Association Information Services.

27. Meyer G, Foster N, Christrup S, Eisenberg J. Setting a research agenda for medical errors and patient safety. Health Serv Res [Internet]. 2001 Apr. [cited 2007 Jan. 9]; 36(1 Pt 1). Disponible en:: http://www.pubmedcentral.nih.gov/picrender.fcgi? artid $=1089210 \&$ blobtype $=$ pdf

28. SIAARTI Study Group for Safety in Anesthesia and Intensive Care et al. Recommendations on the transport of critically ill patient. Minerva Anestesiol [Internet]. 2006 Oct. [cited 2007 Jan. 9]; 72(10): XXXVII-LVII. Disponible en: http://www.minervamedica.it/ index2.t?show $=$ R02Y2006N10A0768F Italian, English.

29. Institute of Medicine. Medicare: A Strategy for Quality Assurance, Volume II. Washington, D.C.: National Academy Press; 1990.

30. Cullen DJ, Bates DW, Small SD, et al. The Incident Reporting System Does Not Detect Adverse Drug Events: A Problem for quality improvement. Jt Comm J Qual Improv. 1995; 21 (10)541-548.

31. Joint Commission on Accreditation of 
Healthcare Organizations. 1998 Hospital. Accreditation Standards. Oakbrook, IL: Joint Commission, 1998.

32. Kohn L, Corrigan J, Donaldson M. To err is human. Building a safer health system. $1^{\text {a }}$ edición. Washington: National AcademyPress; 2000.

33. Maugh TH. $2^{\circ}$ Cancer deaths decline for 1st time since 1930: the drop in U.S. is slight but marks a milestone, as advances catch up with demographics. Los Angeles Times. 2006 Feb 9; Sect. A:6 (col. 1).

34. Ross Baker G, Norton PG, Flintoft V, Blais R, Brown A, Cox J, et al. The Canadian Adverse Events Study: the incidence of adverse events among hospital patients in Canada. CAMJ. 2004; 170: 1678-86.

35. Forster AJ, Asmis TR, Clark HD, Al Saied G, Code CC, Caughey SC, et al. Ottawa Hospital Patient Safety Study: incidence and timing of adverse events in patients admitted to a Canadian teaching hospital. CMAJ. 2004; 170: 1235-40.

36. Woolf S. Patient safety is not enough: targeting quality improvements to optimize the health of the population. Ann Intern Med. 2004; 140: 33-6.

37. Frey B, Buettiker V, Hug MI, Waldvogel K, Gessler P, Ghelfi D, et al. Does critical incident reporting contribute to medication error prevention? Eur J Pediatr.
2002; 161: 594-9.

38. Beckmann U, Bohringer C, Careless R, Gillies DM, Runciman WB, Wu AW, et al. Evaluation of two methods for quality improvement in intensive care: facilitated incident monitoring and retrospective medical chart review. Crit Care Med. 2003; 31:1006-11.

39. Barach P, Berwick DM. Patient safety and the reliability of health care systems. Ann Intern Med. 2003;138: 997.

40. Reason J. Human error: models and management. BMJ. 2000; 320:768-70.

41. Evans SM, Berry JG, Smith BJ, Esterman AJ. Anonimity or transparency in reporting of medical error: a communitybased survey in South Australia. MJA. 2004; 180: 577-80.

42. Carrillo G, Gómez O. Administración segura de medicamentos: un momento de cuidado. Avances en el cuidado de Enfermería. Editorial Universidad Nacional de Colombia. 2010; 234-240.

43. Runciman B, Merry A, Smith AM. Improving patients' safety by gathering information. BMJ. 2001; 323:298.

44. Beyea SC. Patient safety first: A critical partnership- Safety for nurses and patients Association of Operating Room Nurses. AORN Journal 2004; 79(6): 1299. 